Check for updates

Cite this: RSC Adv., 2019, 9, 22740

\title{
Flexible and highly sensitive pressure sensors based on microcrack arrays inspired by scorpions $\dagger$
}

\author{
Junqiu Zhang, ${ }^{\text {ab }}$ Tao Sun, ${ }^{a}$ Linpeng Liu, ${ }^{a}$ Shichao Niu, (D) a Kejun Wang, ${ }^{a}$ \\ Honglie Song, (D) Q Qigang Han, (D) ${ }^{\mathrm{d}}$ Zhiwu Han, (D) *a Luquan Ren ${ }^{\mathrm{a}}$ and Qiao Lin ${ }^{\mathrm{b}}$
}

Recently, there has been tremendous interest in flexible pressure sensors to meet the technological demands of modern society. For practical applications, pressure sensors with high sensitivity at small strains and low detection limits are highly desired. In this paper, inspired by the slit sensillum of the scorpion, a flexible pressure sensor is presented which has regular microcrack arrays and its reversed pattern acts as a tunable contact area of the sensing material microstructures. The template with regular crack arrays generated on the inner surface is fabricated using a solvent-induced swelling method, which provides a simple and economical way to obtain the controllable fabrication of crack arrays without any physical damage to materials. At the same time, the working principle of the bio-inspired pressure sensor is attributed to pressure-dependent variations because of the contact area change between the interlocking polydimethylsiloxane films with the negative and positive patterns of the microcrack arrays. The device shows good performance, with a gauge factor of $27.79 \mathrm{kPa}^{-1}(0-2.4 \mathrm{kPa})$, a short response/recovery time (111/95 ms), a low detectable pressure limit and excellent reproducibility over 3000 cycles. Practical applications, such as the detection of human motion and touch sensing, are then tested in this work, and the results imply that it should have significant potential applications in numerous fields. Note that the reversed pattern of the slit sensillum of the scorpion is explored to enhance the performance of pressure sensors, thus opening a new route for the fabrication of flexible pressure sensors, even wearable electronics, in a cost-effective and scalable manner.

Received 15th May 2019

Accepted 29th June 2019

DOI: $10.1039 / c 9 r a 03663 f$

rsc.li/rsc-advances

\section{Introduction}

With the rapid development of wearable electronics and artificial intelligence, flexible pressure sensors have attracted considerable attention for their potential applications in healthcare monitoring, ${ }^{1-3}$ motion detection, ${ }^{4-6}$ smart robots ${ }^{7,8}$ as well as in highly advanced human-interactive devices. ${ }^{9,10}$ To date, many reported studies have focused on flexible pressure sensors, by utilizing capacitive, ${ }^{\mathbf{1 1 , 1 2}}$ piezoelectric, ${ }^{\mathbf{1 3 , 1 4}}$ organic field-effect transistor, ${ }^{15,16}$ triboelectric, ${ }^{17,18}$ and piezoresistive ${ }^{19,20}$ sensing mechanisms. Among them, piezoresistive sensors, which are composed of substrates and conducting material layers, can transduce the applied pressure or mechanical force

${ }^{a}$ Key Laboratory of Bionic Engineering, Ministry of Education, Jilin University, Changchun 130022, People's Republic of China. E-mail: zwhan@jlu.edu.cn

${ }^{b}$ Department of Mechanical Engineering, Columbia University, 500 West 120th Street, New York, NY 10027, USA

'Department of Engineering Mechanics, Center for Flexible Electronics Technology, Center for Mechanics and Materials, Tsinghua University, Beijing 100084, People's Republic of China

${ }^{d}$ College of Materials Science and Engineering, Jilin University, Changchun 130022, People's Republic of China

$\dagger$ Electronic supplementary information (ESI) available. See DOI: 10.1039/c9ra03663f into a change in resistance, and this has attracted tremendous research interest because of its simple sensing mechanism, easier fabrication, high reproducibility and low energy consumption. ${ }^{21,22}$ For higher performance, flexible substrates and active materials play an important role in developing piezoresistive pressure sensors.

Generally, the selection of an outstanding active material is one of the most predominant approaches to obtaining pressure sensors with a high sensitivity. To date, materials such as carbon nanotubes (CNTs), ${ }^{23,24}$ carbon black, ${ }^{25}$ graphene (including reduced graphite oxide), ${ }^{10,26,27}$ metal nanowires, ${ }^{11,28}$ metal-organic frameworks, ${ }^{29}$ and conductive polymers ${ }^{30,31}$ are usually used as the sensing elements because of their superior mechanical and electrical properties. Furthermore, elastomeric materials, including silicone-based elastomers, rubbers or epoxy, are often exploited as flexible substrates because of their excellent flexibility, stretchability and stability. However, sensing materials endowed with outstanding electrical properties commonly have an undesired impact on the performance of pressure sensors. For example, CNTs can bring high sensitivity and tunable metallic/semiconducting properties. ${ }^{32}$ Nevertheless, the interface between the CNTs and the supporting substrates is not strong enough, which influences the stability and service life of the pressure sensors. Therefore, introducing 
particular engineering structures to polymeric surfaces is another efficient way to realize the performance enhancement of pressure sensors has not been heavily promoted yet. Engineering structures such as pyramid arrays, ${ }^{17,33}$ micropillar arrays, ${ }^{34}$ microgrooves, ${ }^{35}$ interlocking ${ }^{36}$ and aligned $^{37}$ nanofibers, and hierarchical constructions, ${ }^{38}$ have been fabricated to endow flexible pressure sensors with greatly enhanced pressure sensitivity. For example, the sensitivity of a pressure sensor with pyramid arrays was approximately 50 times higher than that of the pressure sensor with no microstructures. ${ }^{33}$ Despite the fact that significant device sensitivity has been achieved, the previously mentioned microstructured films are mainly replicated from silicon molds. The sophisticated molds largely depend on traditional lithography followed by a wet or dry etching process to form the desired geometry, ${ }^{19}$ which is a complicated, highcost and time-consuming process with limited scalability. Thus, it is a great challenge to fabricate pressure sensors with high sensitivity and on larger production scales.

Fortunately, nature often offers inspiration for the advancement of science and technology. Inspired from the silt sensory receptor of arthropods, researchers have established various crack-shaped, wearable strain sensors, which benefit from the connection of the reversible crack fracture surfaces..$^{39}$ In contrast, the crack-shaped structure is scarcely exploited to fabricate flexible pressure sensors because much more control over the crack's morphology and layout is still required to turn the sensor into a sensitive one for pressure sensing. For example, Choi et al. developed a flexible pressure sensor based on guiding straight cracks through a stretched metal layer deposited on polyurethane acrylate (PUA) film. Before deposition, the PUA film was pretreated with ultraviolet flood exposure. ${ }^{40}$ In addition to the complicated and multi-step processes, the crack sensor was mounted on a custom built machine in order to realize deformation to measure pressure, however this operates over a limited sensitivity and measurement regime. In previous work, a bio-inspired strain sensor based on regular microcrack arrays inspired by scorpions was prepared. Wellregulated microcrack arrays were fabricated using the solvent induced swelling and double template transfer method. ${ }^{41}$ However, strain sensors rely on structural deformation to achieve measurements, and so this is not suitable for complex surfaces with a large curvature. Furthermore, most reported strain sensors show a fairly limited sensing range. In addition, reversibly interlocked devices with specially designed physical structures can be found in nature, such as the wing-to-body locking device in beetles. ${ }^{42}$ This interlocking is operated by bringing dense microhairs on the cuticular surface in contact with each other to fix the wings of the insects. In this paper, a new bio-inspired strategy to fabricate flexible sensors with delicately geometric microstructures, inspired from the slit sensillum of scorpions, and its reversed pattern as a intelligently tunable contact area of sensing material microstructures is reported. Based on the crack geometry of the slit sensillum distributed on the legs, which accounts for the excellent vibrational sensing ability of scorpions, regular microcrack arrays and its reversed arrays were fabricated without damage to materials using the same method as used before.$^{41}$ Because of the effective interlocking of the crack arrays and its reversed pattern, the pressure sensor shows ultrahigh sensitivity and great potential for wearable electronic devices as well as in robot sensing systems. As far as is known, the reversed microstructures of the creatures used in the sensor fabrication have not been exploited. It is believed that this unique approach opens a new route to fabricate flexible pressure sensors, and even wearable electronics, in a cost-effective and scalable way.

\section{Experimental section}

\section{Preparation of crack arrays on a PS Petri dish lid}

Crack arrays were generated on the polystyrene (PS) Petri dish lid using the solvent-induced method reported previously. ${ }^{41}$

\section{Preparation of PDMS positive and negative replicas using PS crack array molding}

To fabricate the reversed pattern of the crack arrays, polydimethylsiloxane (PDMS, Dow Corning Sylgard 184), consisting of a pre-polymer and curing agent was manually mixed in the proportion of $10: 1(\mathrm{w} / \mathrm{w})$, and cast onto the Petri dish lid after mixing uniformly. Next the sample was put into a vacuum chamber (BZF-30, Shanghai Boxun Industrial Co., Ltd) for $1 \mathrm{~h}$ to remove air bubbles. After heating in an oven (GZX-GF, LongYue Instrument Equipment Co., Ltd) at $70^{\circ} \mathrm{C}$ for $3 \mathrm{~h}$, the PDMS film with a negative pattern was carefully peeled off from the Petri dish lid. Crack arrays were transferred onto the surface of a PDMS film using a double template transferring method. Epoxy AB glue (Ausbond (China) Co., Ltd) composed of a resin precursor and curing agent, was mixed thoroughly in a $3: 1(\mathrm{w} /$ w) ratio to fabricate an intermediate transition layer and was then poured into the Petri dish lid. After a vacuum degassing process the sample was heated at $60^{\circ} \mathrm{C}$ for $2 \mathrm{~h}$, and then peeled off the Petri dish lid after curing. Next, the PDMS was mixed uniformly in a $10: 1(\mathrm{w} / \mathrm{w})$ ratio, and then cast onto the epoxy mold. A $1 \mathrm{~h}$ degassing process was needed before the sample was put in the oven. After a $3 \mathrm{~h}$ heating process, the PDMS film was peeled off from the solid Epoxy AB film with the crack arrays on the surface.

\section{Fabrication of microcrack array-based pressure sensor}

The microstructured PDMS films were cut into pieces with dimensions of $40 \times 10 \mathrm{~mm}(l \times w)$. Then samples with regular positive and negative patterns of crack arrays were selected and treated $(120 \mathrm{~W}, 20 \mathrm{~s})$ by plasma and then immersed in a sodium lauryl sulfate (SDS, Shengtongxin Company, Tianjin, China) solution $(0.5 \%, 15 \mathrm{~s})$. A $30 \mathrm{~nm}$ thick silver (Ag) layer was deposited on the top of the PDMS sheets to introduce a layer of electricity active using sputter coating (108auto sputter coater, Cressington). After copper $(\mathrm{Cu})$ paste and $\mathrm{Cu}$ wires were anchored on the Ag layer, two layers of coated PDMS films with the micro-structured surfaces were placed face-to-face, to make the final bio-inspired flexible pressure sensors. In order to exclude the interference of environmental factors, the pressure sensor was covered with a $50 \mu \mathrm{m}$ thick encapsulation layer. 


\section{Results and discussion}

Fig. 1 illustrates the novel design of the flexible pressure sensor inspired by scorpions, which were sensitive to environmental vibrations. After undergoing billions of years of natural selection and evolution, a number of creatures have gained the knowledge on how to realize optimal functions with the lowest cost of resources. Microstructures widely exist upon the surfaces of natural creatures. ${ }^{43}$ With the existence of these characteristic surface constructions, plants or animals can have a self-cleaning function, ${ }^{44}$ possess highly dry adhesion, ${ }^{45}$ and exhibit sensitive, stimuli-responsive behaviors. ${ }^{\mathbf{4 6}}$ Thus, researchers have done much research to develop biomimetic functional surfaces to mimic the intrinsic structures of creatures, while showing little interest in the reversed pattern of the micro-structured surfaces of creatures.

As shown in Fig. 1a, it was demonstrated that scorpions are extremely sensitive to vibratory stimuli. Scorpions sense minute vibrations with sensory sensillum called the slit sensillum for the detection of prey and predators, and which are embedded in the cuticle near their leg joints. ${ }^{47}$ In order to characterize the surface morphology of the crack-shaped slit sensillum from Heterometrus petersii (Giant Forest Scorpion), scanning electronic microscopy (SEM) was used (Fig. 1b). It was found that the slit sensillum consisted of $\sim 12$ microcrack units arranged in a radial-like pattern on the surface (Fig. 1c). Inspired by the geometry and the function of the slit sensillum (Fig. S1, ESI $\dagger$ ), a flexible pressure sensor based on microcrack arrays is presented in this paper. The strategy that relies on the utilization of crack arrays inside the active materials, in which charge carrier transport occurs via a tunneling effect has always been regarded as an effective way to fabricate a strain sensor with high sensitivity. However, crack arrays have rarely been explored for the development of pressure sensors because more control over the morphology and the arrangement of the cracks were required to achieve pressure sensing. In addition to the well-controlled crack arrays that were fabricated in this research, it is worth noting that the reversed pattern of the crack-shaped slit sensillum was creatively utilized to achieve reversible interlocking with the crack arrays, which contributes to the extremely high sensitivity to the external pressure applied on the pressure sensor (Fig. 1d). In general, this is a totally new approach to the fabrication of flexible pressure sensors in a simple yet efficient manner.

Fig. 2a illustrates the overall fabrication process for the pressure sensor based on the microcrack arrays. To imitate the geometry of the sensillum, the PS Petri dish acted as the template with regular crack arrays generated on the inner surface using a solvent-induced swelling method reported previously. It provided a simple and economical way to obtain the controllable fabrication of the dimensions of the cracks. ${ }^{\mathbf{4 1}}$ Then, PDMS was selected as the substrate in this study because of its excellent elasticity and its biomedical compliance for use with human tissue. ${ }^{48}$ After a one-step molding from the PS Petri dish with the uniform crack arrays initiated, the PDMS film can be endowed with the negative pattern of the crack arrays, and a double template process was needed to transfer the crack arrays onto the top of the PDMS film. In terms of structure, the fabricated PDMS films achieved a high level of simulation of the morphology of the slit sensillum of the scorpion. The thickness of the PDMS films was controlled in the range of $300 \pm 50 \mu \mathrm{m}$. The morphology and microstructure of the PDMS films with the negative as well as the positive patterns of the crack arrays were observed using atomic force microscopy (AFM, Fig. 2b and c). More details, including the uniformity of the microstructures on PDMS and the PS Petri dish, are shown in Fig. S2-S4 (ESI $\dagger$ ). The results indicated that the width and depth of the negative and positive patterns of the cracks were $\sim 3 \mu \mathrm{m}$ and $\sim 2 \mu \mathrm{m}$, respectively.

After fabricating microstructured PDMS substrates, a thin layer of conducting $\mathrm{Ag}$ was deposited upon the surfaces to effectively transport the electrons. The adhesion between the active layers and the flexible substrates plays a vital role in determining the lifetime of the device. In order to reinforce the interface adhesion, the cured PDMS was treated by plasma and then immersed in sodium lauryl sulfate to deposit $-\mathrm{SO}_{3}{ }^{-}$groups on the surface of the PDMS substrate with the microstructures before depositing the Ag layer. It is mainly thought that this

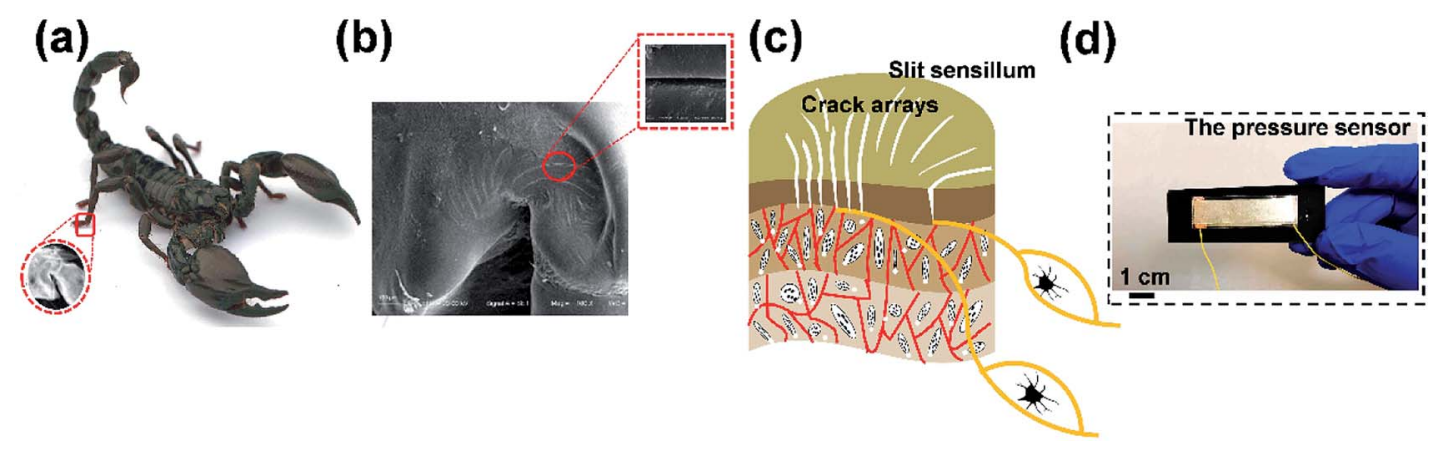

Fig. 1 Schematic illustrations of the pressure sensor based on microcrack arrays inspired by the scorpion. (a) Slit sensillum distributed on the legs of a scorpion for sensing external vibrational signals. The inset is an enlargement showing the accurate position of the slit sensillum, which is located on the distal end of the metatarsus. (b) SEM image of the geometry of the overall slit sensillum. The inset is an SEM image of a single crack. (c) Illustration of the slit sensillum embedded on the exoskeleton obtained from the image in (b). (d) Digital photograph of the fabricated pressure sensor. 

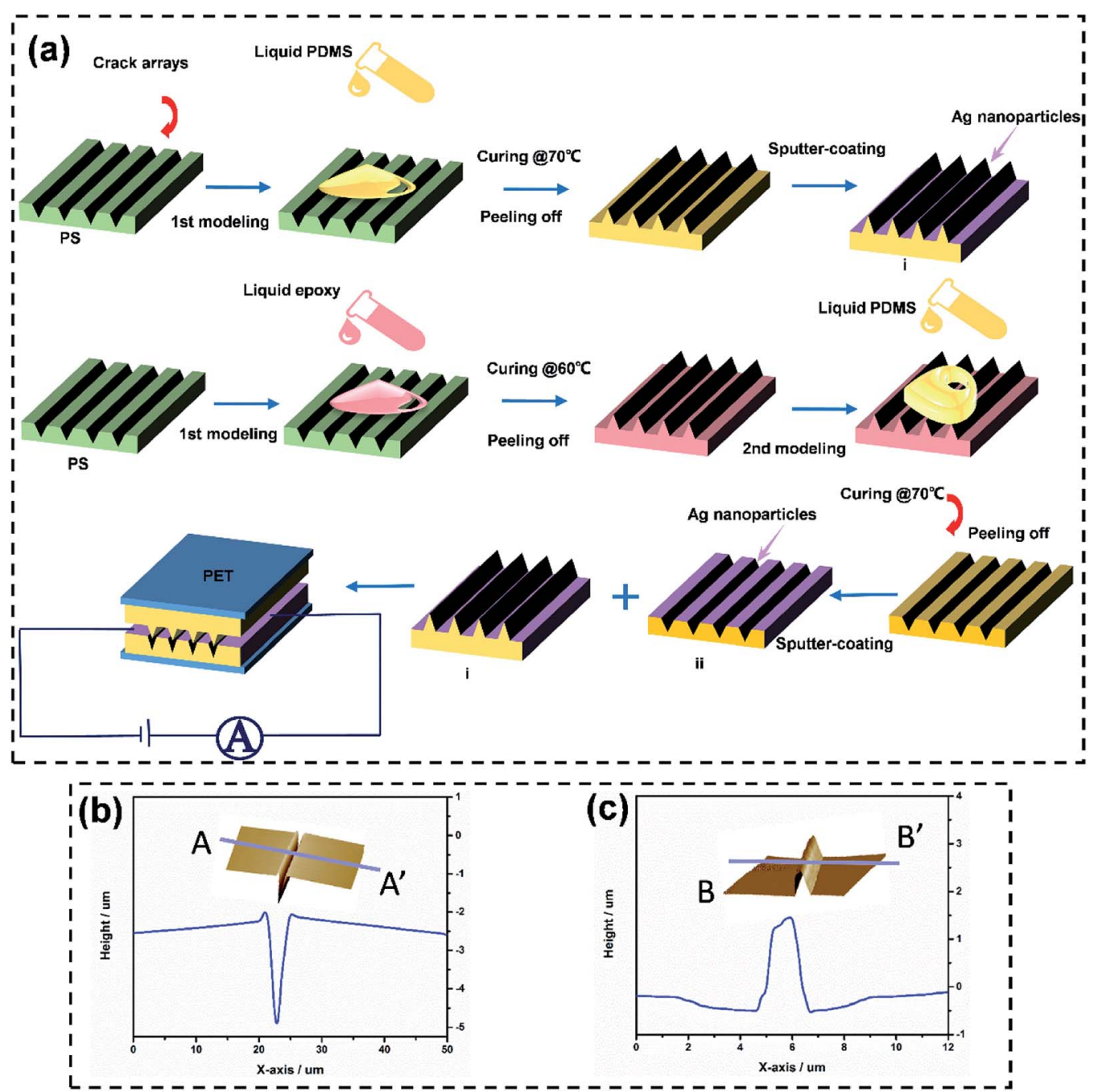

Fig. 2 The fabrication process of the bio-inspired pressure sensor. (a) The negative and positive patterns of microcrack arrays are transferred from the Petri dish lid to the top of the PDMS films. The schematic structure of the pressure sensor has dimensions of $40 \times 10 \mathrm{~mm}(l \times w)$. (b) A cross sectional outline of a single crack measured using AFM. The inset is a 3D image. The width and depth of the crack are $\sim 3 \mu \mathrm{m}$ and $\sim 2 \mu \mathrm{m}$, respectively. (c) A cross sectional outline of a single ridge measured using AFM. The inset is a 3D image. The width and depth of the negative pattern of the crack are $\sim 3 \mu \mathrm{m}$ and $\sim 2 \mu \mathrm{m}$, respectively.

process can introduce a condensation reaction of the hydrophilic functionalities, leading to close contact between PDMS and $\mathrm{Ag}^{+} .^{49}$ Also, in order to avoid the adhesiveness of the PDMS hindering the performance of the pressure sensor, a polyethylene terephthalate (PET) film was placed onto the top and bottom PDMS films as the encapsulation layers.

Fig. 3 shows the working principle of the flexible pressure sensor based on the positive and negative pattern of the crack arrays. Consequently, two layers of coated PDMS films with the microstructured surfaces were placed face-to-face into a logically interlocked construction. Because of the existence of this special construction, external pressure and local deformations concentrated at the small contact spots. As a result, a significant resistance change would appear even at a small external pressure. To better understand the resistive response of the pressure sensor, a simple circuit can be developed, as shown in Fig. 3a. The equivalent circuit diagram could be described as follows:

$$
\begin{gathered}
R=R_{\mathrm{S}}+\frac{n R_{\mathrm{C}}}{{R_{\mathrm{C}}}^{n}} \\
R_{\mathrm{C}}=\left(\frac{2 R_{\mathrm{L}}}{b c}\right) /(b c)^{2}
\end{gathered}
$$

where $R$ stands for the resistance of pressure, $R_{\mathrm{S}}$ is the sum of the resistances of the PDMS surfaces, $R_{\mathrm{C}}$ is the resistance of the contact area between the PDMS films, $R_{\mathrm{L}}$ is the resistance of a single contact interface, $n$ is the number of conducting paths, $b$ is the area of a single contact interface, and $c$ is a constant. Under compressive mode, the sensor response to pressure was reflected in its resistance change with respect to the initial state $\left(R_{0}-R=\Delta R\right)$, as described in eqn (3):

$$
\Delta R=\frac{n \Delta R_{\mathrm{C}}}{\Delta R_{\mathrm{C}}{ }^{n}}=\left(\frac{2 n R_{\mathrm{L}}}{\Delta b c}\right) /\left(\frac{R_{\mathrm{L}}}{\Delta b c}\right)^{2 n}
$$


(a) $\mathrm{Rs}$

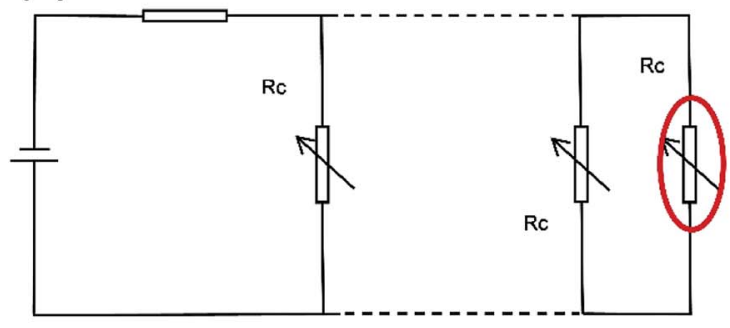

(b)

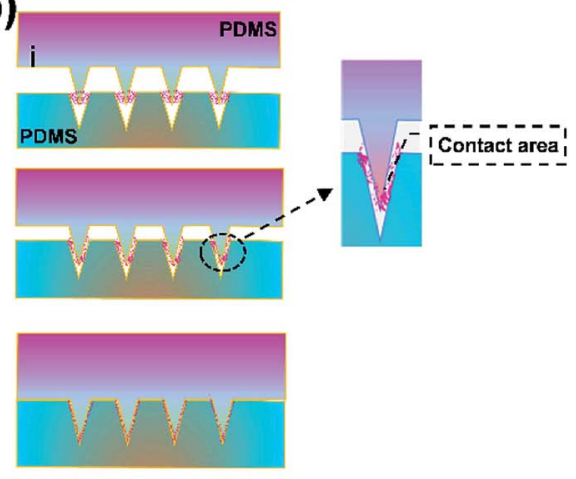

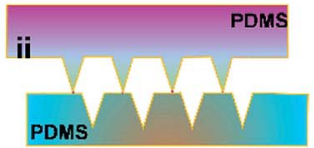

(c)

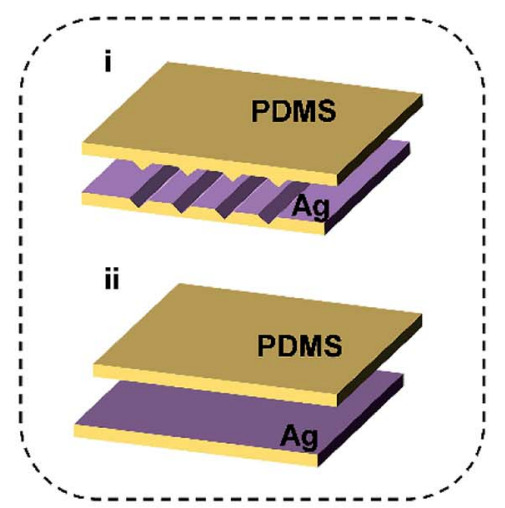

(d)

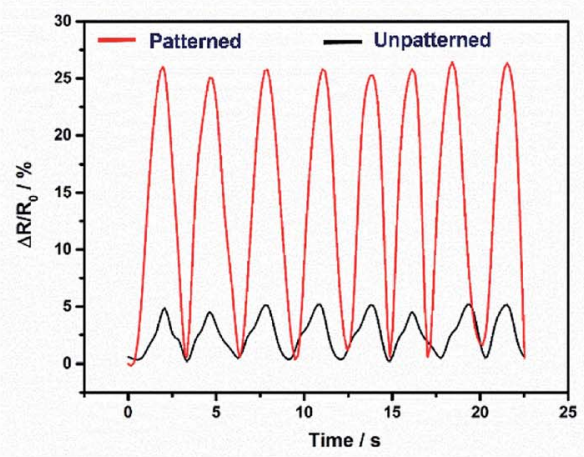

Fig. 3 Working mechanism of the bio-inspired pressure sensor. (a) A schematic illustration of the circuit model. (b) (i) and (ii) An illustration of the operating principle of the sensor under two conditions. The external pressure could yield a resistance decrease and this change would return to the primary state when the external force was removed. (c) The relative resistance variation versus applied pressure for different surface microstructures: (i) the bio-inspired pressure; (ii) the pressure sensor without surface microstructures. (d) The corresponding resistance change versus applied pressure.

where $\Delta b$ is the contact area change, and eqn (3) forms the theoretical basis for quantifying the sensor response to pressure in terms of the contact area change incurred by the top and bottom PDMS films under external force. Thus, a small compression incurred by an external pressure would build a closer interlocking construction, yielding an increased contact area and providing increased conductive as well as a corresponding resistance decrease [Fig. 3b(i)]. After the unloading process, these microstructured PDMS films would recover their original position, resulting in the increase of the resistance to the initial state. Unfortunately, not all the crack arrays and their reversed patterns can form a reversible interlocking construction, as shown in Fig. 3b(ii). When this happens, the reversed pattern on the top PDMS film will deform and yield close contact between the microstructures. Generally, the microstructures on the PDMS surface were beneficial for improving the sensitivity because of the dramatically increased area under a small applied force.

Fig. $3 \mathrm{c}$ and $3 \mathrm{~d}$ also show the outstanding sensitivity of the pressure sensor based on the negative and positive patterns of the crack arrays. To investigate the effect of the microstructures on the sensing performance, the resistance-pressure behaviors of the pressure sensors with and without microstructures were compared, as shown in Fig. 3c(i) and (ii). Under a 350 Pa pressure, the resistance of the pressure sensor with the negative and positive patterns of the crack arrays dramatically decreased whereas the counterpart without a microstructure upon its surface showed a tiny resistance change (Fig. 3d). In the pristine state, the microstructures on the top and bottom of the PDMS films partially contacted and formed a conductive path. When a small pressure was applied, the contact area between the microstructured films can increase significantly, and thus, leads to a more obvious change in resistance than occurs with the unstructured PDMS films, indicating a high sensitivity to the external pressure. The unstructured films show few contacts, leading to a relatively low sensitivity. Furthermore, once the pressure was removed, the resistance returns fast to its initial value, implying that the microstructures can recover quickly. 
The sensitivity $(S)$, which is expressed as $S=\left(R_{0}-R\right) / R_{0} P$, is usually regarded as one of the most important performance parameters for sensors, where $R_{0}$ and $R$ are the initial resistance and resistance under applied pressure, respectively. An ultrahigh sensitivity was obtained by utilizing a compression testing machine (a computer controlled stepping motor and a force sensor). The points in Fig. 4a can be divided into two regions based on the difference in sensitivity. When the applied pressure was less than $2.4 \mathrm{kPa}$ (yellow region), the sensitivity of the device was $27.79 \mathrm{kPa}^{-1}$, which was much higher than that of the relatively large pressure region $\left(1.32 \mathrm{kPa}^{-1}\right.$ in the purple region, 2.4-6 kPa). In brief, a high device sensitivity of $27.79 \mathrm{kPa}^{-1}$ could be generated by this bio-inspired pressure sensor, which was comparable with, or even better, than most reported records of flexible sensors (Table S1, ESI $\dagger$ ). The separated fitting of these points in both regions showed good linear behavior, indicating that the device could be used as a reliable pressure sensor. Here, two different contact modes are applied to explain the changing sensitivity. In the low pressure region, "point contact" was the main contact mode, causing the sharp increase of contact area between the microstructures. After that, the "point contact" gradually evolved into "area contact" with increasing external pressure in the blue region. In general, "area contact" meant that the conductive network became a stable state, and the applied pressure had little effect on the resistance. In short, the conspicuous change in resistance of the interlocked microcrack arrays could be contributed by the great variation in contact area.

To completely evaluate the pressure sensor, other essential performance parameters, including detection limit, stability, reliability and response time, all needed to be considered and examined. Because of the ultrahigh sensitivity of the pressure sensor with the microcrack arrays, it could be suitable for the detection of small changes in pressure. Fig. 4c shows that the pressure sensor can detect a subtle pressure of 9.6 $\mathrm{Pa}$ and $35 \mathrm{~Pa}$ for a pushpin and a corn kernel, respectively, demonstrating the fast response, high sensitivity and reliability of the pressure sensor to a subtle pressure. To investigate the response time of the sensors to applied forces, the output resistance signals were recorded (Fig. 4b). It can be seen that the rise time and drop time were $111 \mathrm{~ms}$ and $95 \mathrm{~ms}$, respectively, indicating that there was a faster resistance signal variation in the force unloading than the force loading process. Theoretically, the response time would be further optimized as the thickness of the PDMS films decrease, because correspondingly it reduced the hysteresis effect from the compression and relaxation times. ${ }^{48}$ Furthermore, the cycling stability of the prepared bio-inspired pressure sensor was measured under a pressure of about $350 \mathrm{~Pa}$. As
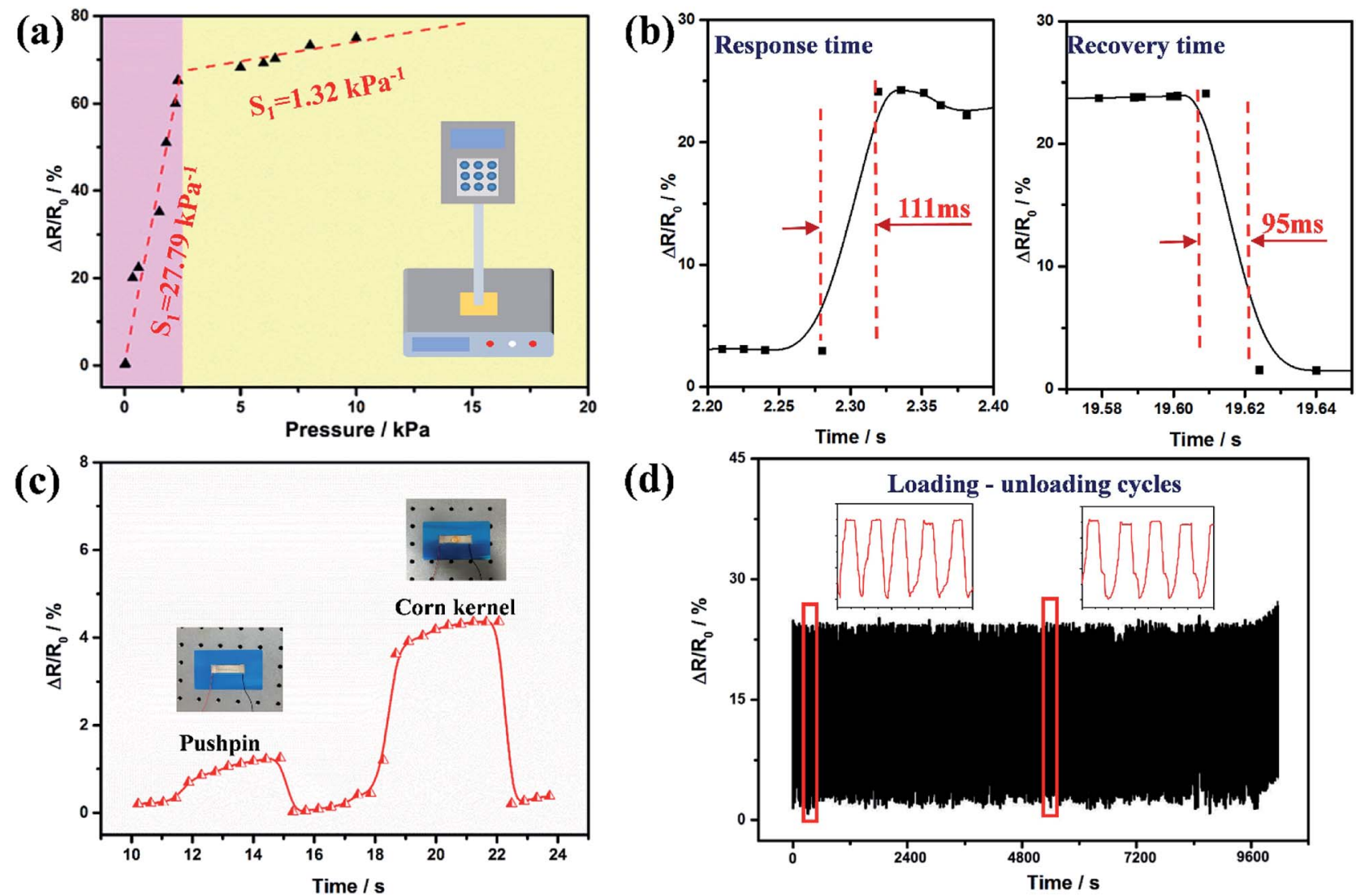

(d)

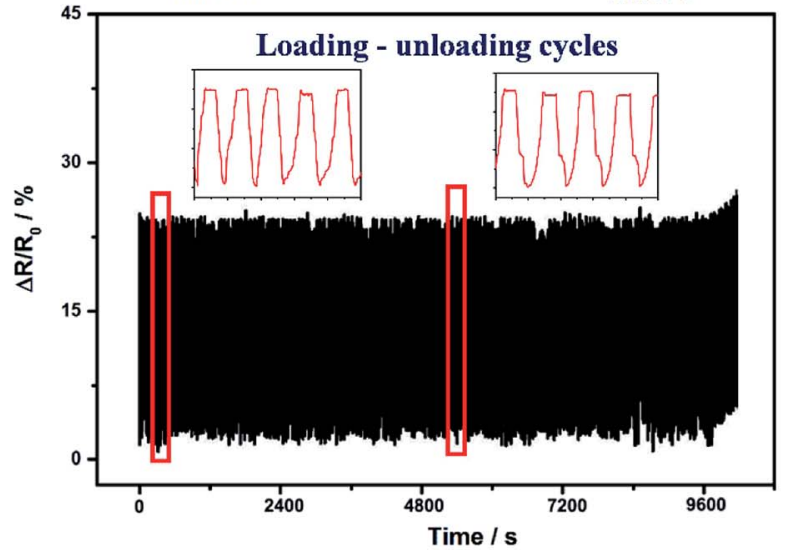

Fig. 4 Performance characterizations of the bio-inspired pressure sensor. (a) The sensitivity of the pressure sensor for pressure in the range of 0-6 kPa. (b) Rise and drop time of the pressure sensor. (c) Relative resistance variation of the pressure sensor under the subtle pressure of a pushpin and a corn kernel loading and unloading. (d) Relative change in resistance under a repeated loading and unloading pressure of 350 Pa for 3000 cycles, which indicates the stability and durability of the pressure sensor. 
(a)

(c)

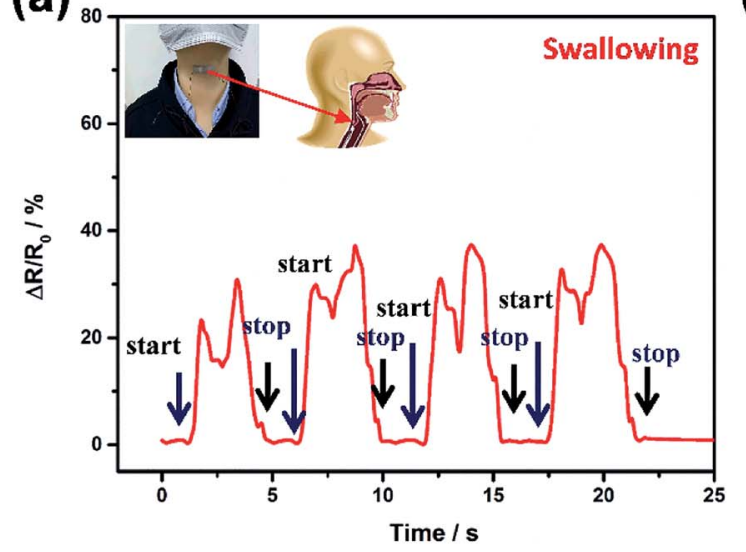

(b)
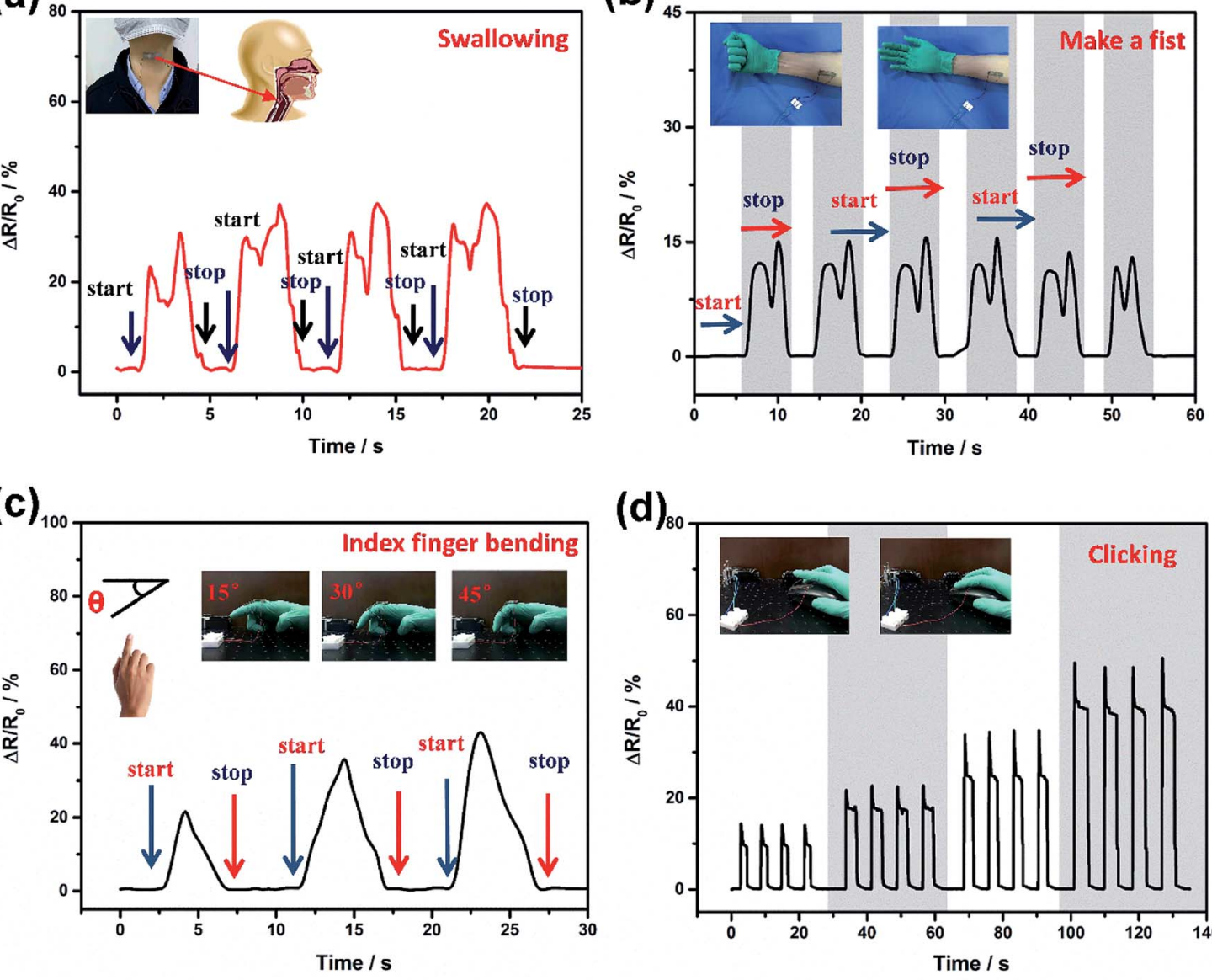

(d)

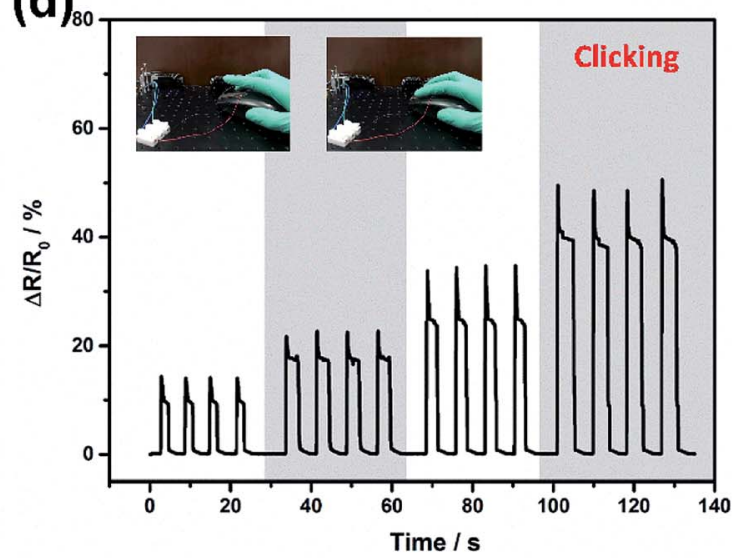

(e)

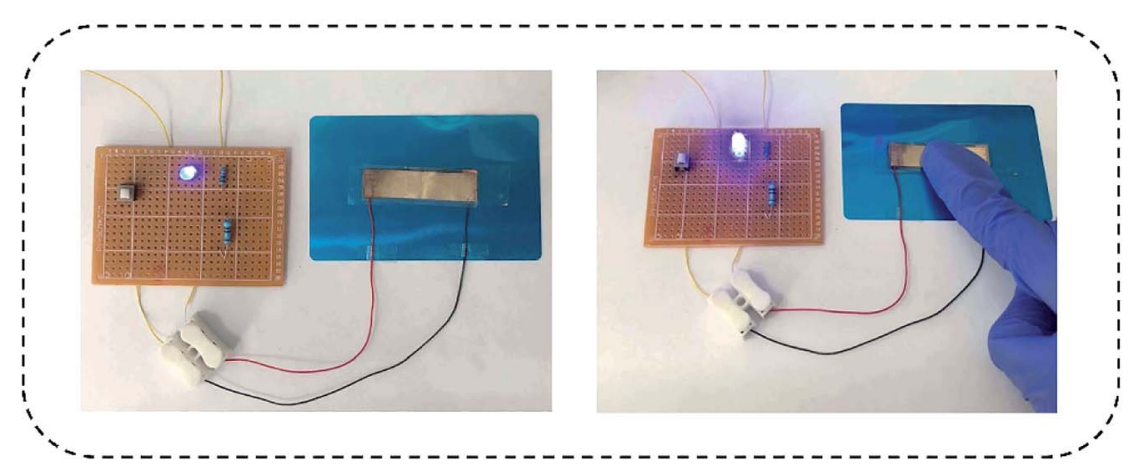

Fig. 5 Applications of the pressure sensor for the detection of various physical signals. (a) Detection of the motion of the human larynx upon swallowing. (b) The sensing performance of wearable pressure sensors mounted on the arm for the reliable detection of radial muscle contraction created by making a fist and opening it. Insets: the sensor adhered on the arm. (c) The sensing repeatability of the sensors attached to a glove-coated finger bent at different angles. Insets: the sensor adhered on the finger. (d) The response of the micro-structured sensor when attached to a PC mouse. The insets show the measurement setup and the simplicity of the flexible sensor. (e) Structure of a simple circuit designed to detect touch and then change the brightness of a LED.

shown in Fig. 4d, there was a repeatable and reliable response and no evident degeneration was observed within loading/ unloading for up to 3000 cycles, implying long working life and reliability of this bio-inspired pressure sensor.

Because of the excellent performance parameters, the bioinspired sensor shows a tremendous potential for use in the detection of human physiological signals and motion activities.
Firstly, the pressure sensor was adhered to a human throat for the real-time and non-invasive sensing of human physiological motion (Fig. 5a). The swallowing of saliva led to the obvious resistance change, indicating the sensor's outstanding ability to distinguish laryngeal motion. This might be helpful in a physiological monitor for the early detection of sudden infant death syndrome (SIDS) in sleeping infants or elderly people, warning 
doctors to any potential problems. ${ }^{41}$ Furthermore, the pressure sensors were mounted onto the forearm for reliable detection of radial muscle contractions caused by making a fist and then reversing it (Fig. 5b). When the tester made a fist, the sensor was under compression, resulting in a decrease in resistance. Also, it showed that the instant sensing performance was reproducible after many cycles. Meanwhile, the bio-inspired sensor was attached onto a finger joint for detecting finger bending at different angles (Fig. 5c). A large resistance change was observed because of the different contact areas between the top and bottom PDMS films. All these results demonstrated that the flexible pressure sensor with negative and positive patterns of microcrack arrays could be used to distinguish subtle amounts of human motion for various potential applications.

In addition, the pressure sensor was attached onto the surface of a PC mouse to detect the gentle tap produced by clicking the PC mouse (Fig. 5d). It was noticed that the sensor had a fast and reliable response to the tap of the finger. When the fingers were taken away, the sensor can return to the initial value. So this experiment suggests that the bio-inspired pressure sensor might have practical applications in flexible touch screens. Further work is in progress to assemble the bioinspired pressure sensors into an active-matrix array in order to simultaneously map the pressure distribution, shape and location of touching sensing. Furthermore, our bio-inspired pressure sensor was used as an action sensitive flexible switch to control the brightness of a light using a self-made circuit (Fig. 5e). When the finger touched and pressed the sensor at a high speed, the pressure sensor showed a prompt and repeatable response with an obvious change in the brightness of the LED (Video, ESI $\dagger$ ). It can be concluded that the bio-inspired pressure sensor had potential for use in highly advanced human-interactive devices and soft robotics as well as in other fields.

\section{Conclusions}

In summary, a bio-inspired flexible pressure sensor was fabricated based on negative and positive patterns of microcrack arrays, which were inspired by the slit sensillum of scorpions. It was noted that the negative and positive patterns of the delicate geometric structure of the slit sensillum were used creatively to build a logical tunable contact area in the sensing material microstructures. Because of the perfectly interlocked constructions, the pressure sensors showed excellent comprehensive performance when compared with other artificial pressure sensors. Also, the uniform and regular microcrack arrays and their reversed pattern were fabricated using a solvent-induced swelling and template transferring method. In addition to the well-controlled morphology and arrangement of the crack arrays, this method could effectively avoid physical damage to the flexible substrates, leading to a long working life, meaning that this was a low-cost, and simple fabrication process. In addition, the importance of the existence of the interlocking of the microcrack arrays and its reversed pattern were emphasized. The bio-inspired pressure sensor showed advantages, such as a high sensitivity of $27.79 \mathrm{kPa}^{-1}$, a quick response/ recovery time (111/95 $\mathrm{ms}$ ) and durable stability (negligible loading-unloading signal changes over 3000 cycles). Thus, the pressure sensor exhibits promising potential for use in flexible wearable electronics and in soft robotics as well as in humaninteractive devices. It is believed that this unique approach will be a new way to fabricate flexible pressure sensors, and even wearable electronics, in a cost-effective and scalable manner.

\section{Conflicts of interest}

There are no conflicts to declare.

\section{Acknowledgements}

This work was supported by the National Natural Science Foundation of China (No. 51675220, 51835006), the Natural Science Foundation of Jilin Province of China (No. 20170101115JC), the JLU Science and Technology Innovative Research Team (No. 2017TD-04), the Science and Technology Research Project of the Education Department of Jilin Province (No. 20190141), the Joint Construction Project of Jilin University and Jilin Province (No. SF2017-3-4), and the China Postdoctoral Science Foundation (No. 2019M650648).

\section{Notes and references}

1 C. Pang, J. H. Koo, A. Nguyen, J. M. Caves, M. G. Kim, A. Chortos, K. Kim, P. J. Wang, J. B. H. Tok and Z. Bao, Adv. Mater., 2015, 27, 634-640.

2 S. Lee, A. Reuveny, J. Reeder, S. Lee, H. Jin, Q. Liu, T. Yokota, T. Sekitani, T. Isoyama, Y. Abe, Z. Suo and T. Someya, Nat. Nanotechnol., 2016, 11, 472-478.

3 Z. Lou, L. Li, L. Wang and G. Shen, Small, 2017, 13, 1-27.

4 T. Q. Trung and N. E. Lee, Adv. Mater., 2016, 28, 4338-4372.

5 X. Wang, Y. Zhang, X. Zhang, Z. Huo, X. Li, M. Que, Z. Peng, H. Wang and C. Pan, Adv. Mater., 2018, 30, 1-8.

6 M. Liao, P. Wan, J. Wen, M. Gong, X. Wu, Y. Wang, R. Shi and L. Zhang, Adv. Funct. Mater., 2017, 27, 1-11.

7 H. Liu, Z. Ji, H. Xu, M. Sun, T. Chen, L. Sun, G. Chen and Z. Wang, Polymers, 2017, 9, 586.

8 L. Wang, D. Chen, K. Jiang and G. Shen, Chem. Soc. Rev., 2017, 46, 6764-6815.

9 Y. Guo, Z. Guo, M. Zhong, P. Wan, W. Zhang and L. Zhang, Small, 2018, 14, 1-9.

10 S. Lim, D. Son, J. Kim, Y. B. Lee, J. K. Song, S. Choi, D. J. Lee, J. H. Kim, M. Lee, T. Hyeon and D. H. Kim, Adv. Funct. Mater., $2015,25,375-383$.

11 X. Shuai, P. Zhu, W. Zeng, Y. Hu, X. Liang, Y. Zhang, R. Sun and C. P. Wong, ACS Appl. Mater. Interfaces, 2017, 9, 2631426324.

12 L. Ma, X. Shuai, Y. Hu, X. Liang, P. Zhu, R. Sun and C. P. Wong, J. Mater. Chem. C, 2018, 6, 13232-13240.

13 W. Wu, X. Wen and Z. L. Wang, Science, 2013, 340, 952-957. 14 C. Dagdeviren, Y. Su, P. Joe, R. Yona, Y. Liu, Y. S. Kim, Y. Huang, A. R. Damadoran, J. Xia, L. W. Martin, Y. Huang and J. A. Rogers, Nat. Commun., 2014, 5, 1-10. 
15 G. Schwartz, B. C.-K. Tee, J. Mei, A. L. Appleton, D. H. Kim, H. Wang and Z. Bao, Nat. Commun., 2013, 4, 1859.

16 Y. Chu, X. Wu, J. Lu, D. Liu, J. Du, G. Zhang and J. Huang, Adv. Sci., 2016, 3, 1-6.

17 W. Yang, J. Chen, X. Wen, Q. Jing, J. Yang, Y. Su, G. Zhu, W. Wu and Z. L. Wang, ACS Appl. Mater. Interfaces, 2014, 6, 7479-7484.

18 L. Lin, Y. Xie, S. Wang, W. Wu, S. Niu, X. Wen and Z. L. Wang, ACS Nano, 2013, 7, 8266-8274.

19 T. Li, L. Chen, X. Yang, X. Chen, Z. Zhang, T. Zhao, X. Li and J. Zhang, J. Mater. Chem. C, 2019, 7, 1022-1027.

20 H. Liu, M. Dong, W. Huang, J. Gao, K. Dai, J. Guo, G. Zheng, C. Liu, C. Shen and Z. Guo, J. Mater. Chem. C, 2017, 5, 73-83.

21 S. Jung, J. H. Kim, J. Kim, S. Choi, J. Lee, I. Park, T. Hyeon and D. H. Kim, Adv. Mater., 2014, 26, 4825-4830.

22 H. Bin Yao, J. Ge, C. F. Wang, X. Wang, W. Hu, Z. J. Zheng, Y. Ni and S. H. Yu, Adv. Mater., 2013, 25, 6692-6698.

23 S. Zhao, J. Li, D. Cao, G. Zhang, J. Li, K. Li, Y. Yang, W. Wang, Y. Jin, R. Sun and C. P. Wong, ACS Appl. Mater. Interfaces, 2017, 9, 12147-12164.

24 H. Chen, Z. Su, Y. Song, X. Cheng, X. Chen, B. Meng, Z. Song, D. Chen and H. Zhang, Adv. Funct. Mater., 2017, 27, 1604434.

25 N. Lu, C. Lu, S. Yang and J. Rogers, Adv. Funct. Mater., 2012, 22, 4044-4050.

26 Y. Cheng, R. Wang, J. Sun and L. Gao, Adv. Mater., 2015, 27, 7365-7371.

27 L. Q. Tao, K. N. Zhang, H. Tian, Y. Liu, D. Y. Wang, Y. Q. Chen, Y. Yang and T. L. Ren, ACS Nano, 2017, 11, 8790-8795.

28 S. Yao and Y. Zhu, Nanoscale, 2014, 6, 2345-2352.

29 X. Fu, H. Dong, Y. Zhen and W. Hu, Small, 2015, 11, 33513356.

30 M. Amjadi, Y. J. Yoon and I. Park, Nanotechnology, 2015, 26, 375501 .

31 W. Zhong, Q. Liu, Y. Wu, Y. Wang, X. Qing, M. Li, K. Liu, W. Wang and D. Wang, Nanoscale, 2016, 8, 12105-12112.

32 P. Lv, K. Yu, X. Tan, R. Zheng, Y. Ni, Z. Wang, C. Liu and W. Wei, RSC Adv., 2016, 6, 11256-11261.
33 C. L. Choong, M. B. Shim, B. S. Lee, S. Jeon, D. S. Ko, T. H. Kang, J. Bae, S. H. Lee, K. E. Byun, J. Im, Y. J. Jeong, C. E. Park, J. J. Park and U. I. Chung, Adv. Mater., 2014, 26, 3451-3458.

34 Q. Shao, Z. Niu, M. Hirtz, L. Jiang, Y. Liu, Z. Wang and X. Chen, Small, 2014, 10, 1466-1472.

35 L. Pan, A. Chortos, G. Yu, Y. Wang, S. Isaacson, R. Allen, Y. Shi, R. Dauskardt and Z. Bao, Nat. Commun., 2014, 5, 1-8. 36 C. Pang, G. Y. Lee, T. Il Kim, S. M. Kim, H. N. Kim, S. H. Ahn and K. Y. Suh, Nat. Mater., 2012, 11, 795-801.

37 Q. Gao, H. Meguro, S. Okamoto and M. Kimura, Langmuir, 2012, 28, 17593-17596.

38 J. Shi, Z. Yang, K. Xia, X. Zhang, H. Li, B. Sun, T. Pan and G. Ding, Flexible Printed Electron., 2018, 4, 015003.

39 D. Kang, P. V. Pikhitsa, Y. W. Choi, C. Lee, S. S. Shin, L. Piao, B. Park, K. Y. Suh, T. Il Kim and M. Choi, Nature, 2014, 516, 222-226.

40 Y. W. Choi, D. Kang, P. V. Pikhitsa, T. Lee, S. M. Kim, G. Lee, D. Tahk and M. Choi, Sci. Rep., 2017, 7, 1-8.

41 Z. Han, L. Liu, J. Zhang, Q. Han, K. Wang, H. Song, Z. Wang, Z. Jiao, S. Niu and L. Ren, Nanoscale, 2018, 10, 15178-15186. 42 C. Pang, T. Kim, W. G. Bae, D. Kang and S. M. Kim, Adv. Mater., 2012, 24, 475-479.

43 Z. Han, Z. Mu, W. Yin, W. Li, S. Niu, J. Zhang and L. Ren, Adv. Colloid Interface Sci., 2016, 234, 27-50.

44 H. Chen, P. Zhang, L. Zhang, H. Liu, Y. Jiang, D. Zhang, Z. Han and L. Jiang, Nature, 2016, 532, 85-89.

45 M. K. Kwak, C. Pang, H. E. Jeong, H. N. Kim, H. Yoon, H. S. Jung and K. Y. Suh, Adv. Funct. Mater., 2011, 21, 3606-3616.

46 E. Gingl, A. M. Burger and F. G. Barth, J. Comp. Physiol., 2006, 192, 551-558.

47 F. G. Barth and M. Wadepuhl, J. Morphol., 1975, 145, 209227.

48 T. Yang, D. Xie, Z. Li and H. Zhu, Mater. Sci. Eng., R, 2017, 115, 1-37.

49 J. Cui, B. Zhang, J. Duan, H. Guo and J. Tang, Sensors, 2016, 16, 2131. 